state of minimum energy. In the case of X-ray and neutron crystallography, the iterative 'shake-and-bake' algorithm ${ }^{1}$ has been revolutionary. This method involves the random perturbation of the positions of atoms in a crystal until the lowest-energy state is found, and it has reduced the time required for determining crystal structures from months to just hours.

Juhás and colleagues call their approach ${ }^{2}$ for inverting PDF data the 'Liga algorithm', because the method is modelled on the rules of promotion and relegation that determine the position of participating teams in most of the world's soccer leagues. Teams correspond to trial clusters of atoms; 'winning' clusters (those with the smallest errors between the model and the experiment) are iteratively promoted, whereas losing ones (those with the largest errors) are relegated, so that an optimal global structure is more quickly found. The authors show that their algorithm can determine a number of nanoscale structures, such as that of the 'buckyball' $\mathrm{C}_{60}$ molecule, with a perfect success rate. Genetic algorithms, in contrast, take considerably longer and have far lower rates of success.

So what are the limits of this approach, and can it be extended to other global-optimization problems? The limits are typically reached when there are more parameters in the theoretical model than can be represented by data, so that the inverse problem becomes 'ill-conditioned' - that is, it has unstable solutions. Optimization strategies must therefore include some way of stabilizing the solutions. Some of these approaches, such as choosing model parameters by guesswork, can involve more than a whiff of the black art, and potentially produce results that vary widely from one investigator to the next.

Alternative methods using powerful statistical methods such as bayesian analysis have been developed, which can avoid the arbitrariness of choosing model parameters ${ }^{5}$. They achieve stability by taking into account a priori information in order to constrain the overall probability distribution for a particular structure. Strategies such as the Liga algorithm could be extended significantly by including known structural information based on a system's physical and chemical properties or knowledge derived from theory and computational materials science. It may well then be possible to resolve heterogeneous nanostructures containing many hundreds of atoms. John J. Rehr is in the Department of Physics, University of Washington, Seattle, Washington 98195-1560, USA.

e-mail: jjr@phys.washington.edu

1. Miller, R. et al. Science 259, 1430-1433 (1993)

2. Juhás, P., Cherba, D. M., Duxbury, P. M., Punch, W. F. \& Billinge, S. J. L. Nature 440, 655-658 (2006).

3. Rehr, J. J. \& Albers, R. Rev. Mod. Phys. 72, 621-654 (2000).

4. Wales, D. J. \& Scheraga, H. A. Science $\mathbf{2 8 5}, \mathbf{1 3 6 8 - 1 3 7 2}$ (1999).

5. Krappe, H. J. \& Rossner, H. H. Phys. Rev. B 66, 184303-184323 (2002).

\section{X-RAY IMAGING Soft focus}

\section{From Wilhelm Conrad}

Röntgen's first snapshot of his wife's hand in 1895, to the security scanner that blows Arnold Schwarzenegger's cover in Total Recall, the use of X-rays to image dense objects has been part of common lore. Franz Pfeiffer and colleagues (Nature Phys. doi:10.1038/nphys265; 2006) now realign the popular view. They use $\mathrm{X}$-rays to generate high-contrast images not only of bone, but also of the soft tissues that surround them. The approach could readily be used to improve the diagnostic power of existing medicalimaging equipment.

Conventional medical X-ray imaging uses the fact that the harder and denser the body tissues are, the more radiation they absorb, and the more contrast they produce on $\mathrm{X}$-ray films. This makes it easy to distinguish bones and other dense bodies, such as tumours, from surrounding tissues. But discerning details of softer tissues from only the contrast in absorption is difficult.

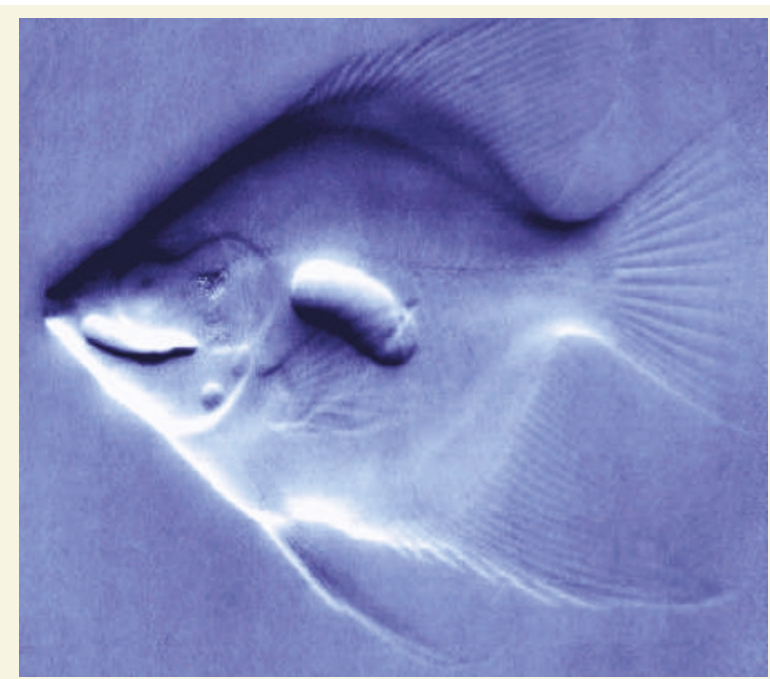

When an X-ray passes through tissue, however, it is not just absorbed: its phase is changed too. And this phase shift is more sensitive to variations in the composition of soft-tissue structures than is absorption. But until now, extracting information about phase has required interferometric reconstruction techniques and bathing the target object in the ultra-highintensity radiation of a synchrotron particle accelerator.

Pfeiffer et al. use a sequence of phase-contrast gratings to manipulate the relative phases of the $X$-rays that illuminate and subsequently emerge from an object. They can thus generate phase-contrast images - for example this $50-\mathrm{mm} \times 50-\mathrm{mm}$ picture of an angelfish - using commercial $X$-ray sources at much lower intensity, and cost, than has previously been possible. The authors note that, as well as improving the detail in X-ray images, their approach could be adapted for use with other lowintensity radiation sources, such as neutrons and ions.

Ed Gerstner

\title{
COGNITIVE SCIENCE
}

\section{Brain development and IQ}

\author{
Richard Passingham
}

\section{If intelligence is partly determined by our genes, how does brain development relate to IQ? An attempt to answer this question measures the size of the outer layer of the brain, the cortex, with surprising results.}

Shaw and colleagues (page 676 of this issue) $)^{1}$ have investigated whether there is a relationship between intelligence and physical dimensions of the brain. Specifically, they measure the thickness of the cortex; the complex computations carried out by the brain depend on the firing of the cortical cells. The authors' results indicate that intelligence can be related to how the cortex changes during development.

Rather than making structural measurements in post-mortem brains, Shaw and colleagues used magnetic resonance imaging (MRI) in living subjects. This allowed the authors to obtain images from people whose IQ could also be tested so as to look for correlations between the two measures. Moreover, detecting anatomical features associated with an individual's intelligence requires a large pool of subjects, because any effects may be small and could be missed if the sample size is inadequate. The use of imaging, rather than post-mortem measurements, allows data to be gathered from a sufficient number of individuals.

The authors scanned 307 children from the age of six years and followed them through adolescence with further scans. For each child, the authors estimated intelligence using subtests of the Wechsler Intelligence Scales - the most commonly used IQ tests. An alternative approach would have been to look at a crosssectional sample of children and adolescents of different ages, scanned only once each. But, as the authors note, such methods are open to many objections: for example, teaching 